Article

\title{
Capturing the City's Heritage On-the-Go: Design Requirements for Mobile Crowdsourced Cultural Heritage
}

\author{
Bas Hannewijk ${ }^{1}$, Federica Lucia Vinella ${ }^{1, *}$, Vassilis-Javed Khan ${ }^{2}$, Ioanna Lykourentzou ${ }^{1}$, \\ Konstantinos Papangelis ${ }^{3}$ and Judith Masthoff ${ }^{1}$ \\ 1 Department of Information Science, Human Centered Computing, Utrecht University, 3584 CC Utrecht, \\ The Netherlands; bashannewijk@live.nl (B.H.); i.lykourentzou@uu.nl (I.L.); j.f.m.masthoff@uu.nl (J.M.) \\ 2 Department of Industrial Design, Systemic Change, Eindhoven University of Technology, \\ 5612 AZ Eindhoven, The Netherlands; v.j.khan@tue.nl \\ 3 School of Interactive Games and Media, Golisano College of Computing and Information Sciences, \\ Rochester Institute of Technology, Rochester, NY 14623, USA; kxpigm@rit.edu \\ * Correspondence: f.l.vinella@uu.nl
}

Received: 27 January 2020; Accepted: 12 March 2020; Published: 20 March 2020

\begin{abstract}
Intangible Cultural Heritage is at a continuous risk of extinction. Where historical artefacts engine the machinery of intercontinental mass-tourism, socio-technical changes are reshaping the anthropomorphic landscapes everywhere on the globe, at an unprecedented rate. There is an increasing urge to tap into the hidden semantics and the anecdotes surrounding people, memories and places. The vast cultural knowledge made of testimony, oral history and traditions constitutes a rich cultural ontology tying together human beings, times, and situations. Altogether, these complex, multidimensional features make the task of data-mapping of intangible cultural heritage a problem of sustainability and preservation. This paper addresses a suggested route for conceiving, designing and appraising a digital framework intended to support the conservation of the intangible experience, from a user and a collective-centred perspective. The framework is designed to help capture the intangible cultural value of all places exhibiting cultural-historical significance, supported by an extensive analysis of the literature. We present a set of design recommendations for designing mobile apps that are intended to converge crowdsourcing to Intangible Cultural Heritage.
\end{abstract}

Keywords: intangible cultural heritage; sustainability; mobile crowdsourcing

\section{Introduction}

The many intangible objects that are part of a city's cultural heritage compose what is known as Intangible Cultural Heritage (ICH) [1]. ICH goes beyond factual aspects of a city's relics. It extends to and includes citizens' memories, thoughts, stories and events that have occurred at a specific location in time. The lack of physical substance is what makes an intangible object less visible and identifiable than a material one. The digital documentation of these abstract stories offers an optimal solution to the intent of recording what would be at risk to be lost from collective memory [2]. The ubiquitous nature of mobile technology [3], e.g., in the form of smartphones, makes designing mobile applications that capture and document novel and old information, a rather attractive solution [4].

Before ICH can be disseminated to a network of users, it needs to be uploaded by the users themselves. One of the crucial aspects for an application to be sustainable and attractive is the embedded capability of attracting new users and retaining existing ones [5]. An ideal ICH framework would be effective at both acquiring and retaining users by intrinsically motivating them in a 
continuous way. In this paper we present an extensive analysis of the following mobile design guidelines and research questions for ICH:

- Which geo-located technological features can help us capture intangible cultural heritage?

- How can people be best motivated to use these features and contribute towards new and existing ICH content?

\section{What Is Intangible Cultural Heritage (ICH)?}

The term Intangible Cultural Heritage was first defined [1] as:

"The practices, representations, expressions, knowledge, skills—as well as the instruments, objects, artefacts and cultural spaces associated therewith-that communities, groups and, in some cases, individuals recognize as part of their cultural heritage. This intangible cultural heritage, transmitted from generation to generation, is constantly recreated by communities and groups in response to their environment, their interaction with nature and their history, and provides them with a sense of identity and continuity, thus promoting respect for cultural diversity and human creativity" [1].

$\mathrm{ICH}$ is a concept consisting of three interdependent and intertwined parts of equal weight. The term intangible, meaning the entity that cannot be touched, refers to people's memories, stories, activities which occurred at a certain point in time, and/or at specific places, including the individuals linked to those memories, stories, and geographical areas. Often, these places correspond to monuments, groups of buildings and sites that are considered tangible [6], but have a cultural value that extends beyond the tangible as it connects meaningfully narratives about history, culture, and identity [7]. This means that intangible cultural heritage is tightly coupled with tangible places as it encompasses the dynamic character which can help promote the social and functional diversity that exists in the historic fabric [8,9]. Deciding on the values associated with the historic fabric, so what is important to preserve, needs to involve the community, as they are best placed to understand what is important [9] (see also [9] for a process for determining the significance of historic fabric). It should be noted that the terms "cultural" and "culture" refer in the context of this work to all aspects of culture, being not only the arts, sciences and languages but also all the traditions leading to different lifestyles [10]. The main reason that culture is better classified as intangible is that it cannot be linked to its tangible products, as it is continuously living and evolving [11]. Heritage can best be described as a property that is or may be inherited. This means that time is also an important factor to include when thinking about intangible cultural heritage. ICH is fluid, different, and is never performed identically [12].

\section{The Importance of Preserving ICH}

Tangible objects, such as buildings and monuments, can be documented and preserved with much more ease than stories and memories, primarily due to their intrinsic physicality. Intangible heritage, on the other hand, lacks this luxury, and is thus more challenging to record, conserve and safeguard [13]. Intangible heritage carries within itself the types of practices and traditions that gave value, meaning and configuration to the associated tangible places. If there is no way to protect this intangible heritage, it risks, for the great part, to go extinguished. Observing the order of investments reserved to the cultural preservation by the International Cultural Organisations, the prevalent trend has been to put, above all, the safekeeping of the tangible objects. Objects that are coupled explicitly with the humanity's visible heritage. Despite the efforts, the world's intangible heritage has received insufficient attention, and the dangers of failing to catalog such knowledge, are so far, of underestimated consequences [14]. Missing at documenting considerable portions of ICH would mean failing at documenting the mainspring of humanity's cultural diversity [15]. UNESCO considers the safeguarding of cultural diversity, consisting of both tangible and intangible heritage, one of its major tasks, as: 'cultural diversity is what makes our world rich and vital' [14]. 
One of the fundamental threats to the sustainability of $\mathrm{ICH}$, perceived by the communities, is largely singled out to be the processes of globalization and development [16]. These advancements have been discussed to significantly threaten cultural heritage resources, not only from a physical point of view, but also in terms of transforming the socio-economic dynamics, and consequently intangible features of the cultural landscape [17]. Globalization and development are hardly avoidable and are regarded by the collective wisdom as the most prominent attacks against the protection of traditional expressions and local cultures [18]. Documenting ICH in the 21th century is a pursuit often challenged by the expansion of the intermingling of cultures, a trend substantially attributed to the effects of globalisation. More so, technology has had a fair share of criticism when looking at the way traditions and oral culture are propagated across generations. With the prospect of the human race, moving towards an even more changeable and uncertain future, collecting the intangible products of these checkpoints in time becomes a pivotal facet of the history of humanity [15]. In spite of globalization and development, political shifts and policy decision-making entities have proven to impact cultures, making the forward march of progress not the only factor at play. Authoritarian governments, for instance, at different levels and degrees of influence, have been pointed at as determinant factors in either the conservation or the shunning of ICH [16]. Present-day is a time where the loss of this diversity and cultural identity plays a crucial role, as well as a time where intangible cultural heritage is threatened by the dispersal of communities, making its protection critical [19]. The safeguarding of these cultures including all of their practices, knowledge, skills, artistic expressions, craftsmanship, dance and performance arts responds to the need of strengthening cultural diversity [19].

Another reason why preserving ICH is important [1] is that it is expected to stimulate international solidarity and cooperation towards the safeguarding of cultural differences and identities [20]. In order for the actions stated above to exhibit, the element of awareness needs to be encouraged towards the recognition of the cultural values of the people involved in the process of cultural holding. One of the first steps that can be taken for achieving greater cultural awareness, is to reach out to the families as well as the educational institutions which play the roles of knowledge buffers between the individual and the society [21]. An application that can help capturing the ICH of a place, could swiftly bridge the gaps between those cultures at risk of extinction and the appropriate cultural institutions, whilst placing the user at the center of the preservation process.

So, safeguarding intangible cultural heritage is extremely important. ICH strongly connects with cultural diversity, susceptible to technological developments and globalization. We suggest to initiate this process by starting from an understanding of the difficulties faced when documenting $\mathrm{ICH}$, and instigating the necessary awareness regarding this problem. An application that captures ICH can create a safe zone from where the act of understanding cultural diversity and the growth of personal sensitivity towards the heterogeneity of $\mathrm{ICH}$ can together develop into practices that are widely taken up and encouraged.

\section{Existing Ways of Safeguarding ICH}

Before even considering ways to safeguard $\mathrm{ICH}$, it is important to mention that in the majority of countries around the globe, it is impossible to protect communities' property rights as there are no legal provisions, and that communities may be unaware of their heritage's potential value to others [22]. There have been attempts to protect and preserve the world's cultural heritage in the past. Japan, for instance, through the endorsement of the Law for the Protection of Cultural Properties [23], has listed a set of both tangible and intangible properties, including humans, as living treasures. All of this with the aim to preserve the Japanese ICH. The consequences of a failure to comply with this law have been treated by the government at the same level of importance as any malicious attacks carried against the very survival of Japanese civilization. Other countries such as South Korea, the Philippines, France, and Romania have all set up similar programs in response to similar concerns [24]. 
Other deliberate efforts towards the protection and preservation of $\mathrm{ICH}$ were discussed in meetings held in the 1980s by UNESCO (such as setting up educational programmes, special science funding for the preservation of folklore, dedicated (sections in) museums), however, very few countries have used these since [24].

The International Convention for the Safeguarding of the Intangible Cultural Heritage [25] has been the first intercontinental attempt to standardize the conservation of ICH with a digital plan of action. The primary objective being to urge nations to develop a digital inventory consisting of all intangible information related to culture, with the underlying contributions of groups and communities [20]. Despite most nations efforts to abide with the call, the challenge of documenting and preserving ICH remains an open issue [24]. The concept of crowdsourcing in the digital area can soon become the imperative source for collecting and managing ICH databases, especially with the convenience of all-in one mobile applications [6].

As a result, more and more nations are investigating ways to digitize their collections [24] to make them accessible to the public. In India, for instance, the governmental announcement to carry out comprehensive documentation of all of its national intangible heritage ensures that every expression of heritage will get a spot in the preservation. The Indian government wants to accomplish this by making extensive digital inventories to store ICH data for the future [26]. An application relying upon user-generated content could help to digitize and catalog ICH data for wide access and usage, more conveniently than analogical means.

ICH can also be considered Intellectual Property. This domain-shift sees ICH turning into the subject of an individualistic approach, identifying individuals as the creators and guarantors of the intangible. Treating ICH as Intellectual Property opens ways to attribute its content to copyrights, patents, trademarks, etc., thus protecting the holders and the data. Looking at the entirety of the information ecosystem, heritage protection has mostly been done using similar approaches [26]. Nevertheless, the limits are still present [27]. Firstly, it is hard to identify a single creator of ICH that is passed down generations whilst being shared within the community. Secondly, ICH outlives its creators, questioning the concept of ownership. Thirdly, transforming non-material heritage to tangible could potentially inhibit permutations of the same [19]. This approach also risks privatizing something that belongs to a community instead of a specific person [26]. The restrictions coming from the individualistic attribution of ICH are extended, leading to ICH as a community-based approach.

Communities have proven to be vital in enriching cultural diversity [1]. This approach considers intangible cultural heritage more as a knowledge commons, meaning it is a resource that is shared by a group of people comprising communities. Inventorying heritage to its holders plays a big role in safeguarding community ICH, as long as policies are put in place for the prevention and deprecation of any discriminatory misconduct [19].

Safeguard and transmission complete the backbone structure of a sustainable ICH framework. Transmission can be in the form of teaching or training. Teaching allows for acquiring the most important skills and knowledge related to a specific intangible heritage, but does not cover the tacit knowledge. In other cases, intangible knowledge cannot even be taught at all. To overcome these problems, projects should be developed to answer educational needs and make it possible to transfer the knowledge commons to future generations [19]. Capturing and protecting intangible cultural heritage by using a mobile application makes it possible to store and transfer ICH to future generations.

As a concluding remark it should be mentioned that even though technology has the potential to significantly help the preservation of ICH it cannot alleviate most issues surrounding the preservation of ICH without favorable regulatory systems reflecting local conditions and including legislative and regulatory measures aimed at the conservation and management of the intangible attributes of intangible heritage, including its social, environmental, and cultural values. 


\section{General Principles}

Within the bounds of the complexity and fragility of $\mathrm{ICH}$, there are essential questions that need to be addressed. For a start, there is a need to discuss what it means to preserve ICH (Does it mean recording ICH or keeping traditions alive?), what regulations are needed around the process of ICH data collection (Can anyone provide ICH knowledge without any sort of verification?), acquisition (What data is permissible to collect?), handling and storage (What happens to the data once is collected? Who can access it and make changes? How do we prevent misuse?). Additionally, there is a need for ICH data standardisation and the call for a legal framework in the attempt to preserve and validate it, as well as the rights of the people and communities whose ICH is being recorded. Here we address some of these questions in the form of general principles.

\subsection{What It Means to Preserve ICH}

As stated by Bonn et al. [28]: One of the fundamental truths recognized by the living history centers is that much of what we regard as intangible cultural heritage takes the form of embodied practice, and that preservation of heritage requires sustained and repeated enactment of that practice as part of the means by which it is preserved over time. Preserving ICH means maintaining cultural diversity, promoting the understanding of differences and facilitating intercultural dialogue. ICH is not simply a cultural manifestation, but a great wealth of knowledge that is community-based, representative, inclusive, traditional, contemporary and living at the same time [24]. The conservation of ICH can take different forms, based on whether the scope is to preserve it at a certain state, to re-discover it after periods of neglect or keep it alive in the community or through practices and costumes. The choice of what can and cannot be recorded and/or maintained as a living tradition is up to the users and the community due to moral and ethical considerations and the breadth of ICH. Some ICH is better only recorded, as old traditions may no longer by ethical or viable, whilst for other ICH continued practice (which may include evolvement) is important. For example, the Sinterklaas tradition in the Netherlands is part of its ICH, but due to racism concerns the use of Black Petes is currently evolving into a more acceptable form.

\subsection{ICH Privacy Issues for Individuals and the Community}

The privacy concerns arising from data-collecting electronic devices have been analysed in previous research [29-32]. Several international regulations have already been put in place, attempting to solve the digital world's privacy problems, such as the European GDPR regulation that are directly relevant to privacy issues for ICH [33]. The impact of regulations for the reinforcement of privacy of Intellectual property in the context of ICH has been subject of prior studies [34]. The topic of privacy and security is critical to the engineering of any framework designed in the digital age and which, by itself, deserves a designated part of the theoretical groundwork. More related work on this topic for ICH can be found in [19,35-38].

\section{ICH content validity and data integrity}

Data handling can be regulated through either Institutional interventions [39], Wiki Verifiability measures [40], framework-dependent quality assurance sourcing policies [41], dual domain watermarking [42], cataloguing templates [43] etc. The choice of data validity methodologies for the prevention of the 'trivialisation' or 'dumbing down' of the data for ICH collection will depend on the objectives and the scopes of the ICH platform. A commercial mobile application will have to comply with the international regulations concerning data handling. Further preventive methods for reinforcing and preserving data integrity will be an essential part of the engineering of the ICH software, in respect to the content validity and the sustainability of the system.

Content and Images rights-The question of ownership 
Whether the ICH is gathered collectively or via individual contributions, the question of ownership touches all aspects of ICH. A neat differentiation between the notion of "culture" and the one of 'heritage' is presented by the work of Xiao et al. [44], where culture is local and heritage is publicly owned. This differentiation might be at times too generic to fit all of the content ownership-attribution scenarios. Given that the existing legal arrangements concerning heritage remain under the control and power of the Nations, there is still a lot that can be done to give credit to the distinct Indigenous nations that own, enact and assert these heritages in specific cultural terms [12]. The same applies to individuals or small groups of individuals. The question whether labels such as "authenticity", "preservation" and "ownership" should be even applied to the ICH domain is still open. The work by Lixinski offers a critical interpretation of the notion of "ownership" since it argues that there is no such thing as "authentic" intangible heritage, and that to think of intangible heritage in these terms could lead to a dangerous commodification of what should be manifestations of living, constantly-evolving cultures [45]. The debate is indeed still open, and the direction that will be taken by the policy-makers will determine the future of $\mathrm{ICH}$ as a commodity limited by its territorial idiosyncrasy, or better, as common heritage of mankind for the enhancement of cross-cultural dialogue.

\section{The pros and cons about the Digital Recording of ICH}

Converting the 'invisible' to 'visible' with a highly dynamic degree and the full-sensory experience is just one of the known challenges for the digitisation of ICH [46]. With the democratization of the $\mathrm{ICH}$ sourcing there is also the growing risk associated with the lowering of standards. A number of strategies have been documented to face these risks: (a) To note the difference between documentary and video archives; (b) To focus on the selection of digital objects, without blind digitization; (c) To protect the inheritors' rights and data security; (d) To carefully select the digital preservation format and medium; (e) To strengthen the combination with related knowledge and technologies. More about these can be found in the work of Yang [46].

\section{Ethical and Moral principles of ICH}

The first point closely regards one of the main criticisms that have affected ethnographic and anthropology since the beginning of the concept 'going native' under the point of view of the professional researcher [47]. With the additional introduction of the crowd as source of knowledge, the ethical and epistemological risks associated with the observers not being external and distant in relation to the action recorded is even more so prevalent. The case for the data-providing users that should or should not partake in the unfolding of the ICH can be subject to discussion when designing the application. Another issue is if and how a clear distinction between the observer and the observable should even be considered. The other main problem originating from the recording of $\mathrm{ICH}$ is the requirement of human consent to do so. Ideally, all people documented through a mobile application should be protected at the highest ethical standards with ethical approval forms, however, when recording a big cultural event this may not be possible. In any ICH capture, participants' rights would ideally apply, including the right to be informed about the capture, the right to fully decide whether to participate in the capture, and the right to withdraw at any time without penalty [48].

\section{Data handling and storage-Transparency and purpose}

While most people are broadly aware that companies collect data on them, they are surprisingly uniformed about the specific types of data they give up when they go online [49]. An ICH application that teaches its users about data transparency and handling, allows them to have full control over what is stored and the clear ways that this information is administered and collected would comply with the design recommendations we have herein explained. Users' trust should be at the center of the application design, as voluntarily identifying and adopting the most stringent data privacy policies can, in the long run, not only gain the trust of the users, but also prevent misuses and irregularities. 


\section{Mobile Apps for Capturing ICH}

While many projects exist that try to capture and preserve $\mathrm{ICH}$, not a lot of them try to do this using mobile technologies. Building a platform/system to capture ICH conveniently offers a virtually unlimited access to content resources. However, an examination of the projects made it clear that most solely focus on documentation [50]. Almost all of the projects that actually make use of an app, use the same technology and methods to some extent. Most of the time the user's location, interests and previous choices are used to present intangible cultural information about tangible places and to give suggestions on where to go next. The presentation of this information is mostly done using Augmented Reality. This does not necessarily mean that every app uses the smartphone camera to point in a certain direction, and overlap that view with layers of visual information to mix it with reality [51], but also that geographical positioning data, video and/or sound can be used to transfer information. By taking advantage of Augmented Reality, the user's experience can be enriched by giving him or her a more complete understanding about the cultural content of a specific location or place [52]. There are many developments in the Augmented Reality area with regards to cultural heritage, as mobile app designers are using it to offer experiences that are unique for every user in natural environments [53]. By providing these experiences, they hope that their digital applications will promote more understanding and knowledge about heritage, whilst also creating value and appreciation for it [53].

A good example is a mobile application made for Castello, a neighborhood in Cagliari, Italy. The goal of the app is to guide tourists around the neighborhood, tell them which cultural heritage sites are worth visiting and if there is enough time to do so. This is done using a simple query based on the user's demographics, such as age and physical ability. The app presents the user a list of site recommendations to visit according to thematic and geographic proximity [54]. These lists are connected to multiple "points of interest" (POIs) and "smart walks". These smart walks are routes to a given place with several things to see on the way there. The app calculates the time to get there and also places the POI on a 'smart walk', or route, with more POIs. Once a user has arrived at a POI, the app presents information, guidance, and info obtained about it [51]. The user walks from a start point to a finish point, with several POIs on the way. This also allows for the intangible cultural heritage that is linked to the place to be disseminated [54]. After a visit, the user can rate each visited POI in terms of the quality of service offered. Users can also comment, tag, upload images, add folksonomies (certain taxonomies about a physical place used by the community) and new knowledge about the POI, which might be a landmark [54]. This way every time a POI is visited, more intangible information about it will become available for the next visitor. Another user can decide to take this or another route based on this information. All of this feedback can then be shared on social media, thus reaching more potential visitors. This system can be used by moderators and people that just use the app to find out more about the city. The moderators can improve the application by using feedback from general users and these users can, in turn, enrich the information originally posted by the moderators [51]. This method ensures the broad participation and sharing of information between all actors and creates a balance between material cultural heritage and local wisdom [55].

There are several similar apps as the aforementioned, which add salient features for developing a sustainable platform to capture ICH. For example, members of a local community can also be motivated to use such apps to upload data with authentic intangible cultural heritage information and pass it on to younger generations and people around the world [56].

In another app [52], the designers put the focus on sound. More specifically, the app leverages 'Storytelling' to transform the mobile device into a virtual narrator that tells stories and anecdotes about a place that can enrich the visit, or simply provide the user with historical and social information [57]. This narration can be done using material stored in sound archives tied to a particular location, creating a guided tour based on a GPS location and the points with audio content attached to them. The material can be roughly subdivided into two categories: narratives and excerpts. Narratives are recordings of inhabitants telling their own stories, memories, anecdotes, and oddities they know 
related to the place that would be forgotten were it not recorded. Excerpts includes poetry, theatre, songs, folk tales and popular knowledge belonging to the tangible heritage. As the position of the user alongside the route is constantly logged, users can also create audio files themselves for other people walking that same route to hear later on. In other words, using an audio guide telling authentic stories and information originally recorded in the same area about tangible heritage allows tourists to have access to intangible heritage and look up to the sites, monuments and buildings, without forcing them to look at the screen of their devices [52]. Authorities are increasingly interested in mobile applications that track how people use the city, such as the application used by Florence to track tourist flows [58], and such applications could be expanded to allow the capturing and passing on of $\mathrm{ICH}$.

\section{Towards Capturing ICH through Location-Based Crowdsourcing}

As all data gathering and capturing is actively done by users, our crowd-sourced data falls into the category of non-framework active crowd-sourced geographic information. The following provides a short summary of the tasks one can use in such an app:

- Imaging can be used to ask users to take and capture images of specific POIs.

- Geo-referencing can be used to link these images to coordinates on the map that will be used in the application. The app will do this automatically, or the user can do it manually if he or she thinks the coordinates that the app calculated are not accurate enough.

- Contextualization can be used to attribute information to these POIs. This information can include users' stories, memories, folk tales, traditional songs, thoughts, and more. These can be uploaded using text or audio.

- Validation can be used to assess the quality of the contributions. Users can do this by voting on other people's contributions and moderators can check these assessments.

- Sharing can be used to share contributions and other information in the app with other people. This way people get invited to also use the app and this may attract more users.

\section{Assessing the Quality of Crowd-Sourced Geo-Spatial Data}

The quality of information is one of the crucial points for whether the city application should adopt crowdsourcing and other related methods. Numerous traditional quality factors have been designed to determine whether a piece of data is of sufficient quality to use. These factors include accuracy, lineage, completeness, consistency, temporality, reliability, robustness, truthfulness and credibility [59]. To shortly present each:

- Lineage refers to knowing who uploaded what. This could be solved by implementing a system where users need to create an account with information about them, such as characteristics and interests. This way you always know who contributed what and at the same time the account can be used for other functionalities.

- Attribute accuracy refers to the terminology and classification being used. The app could provide the user with templates he or she needs to fill out when creating a Point of Interest (POI).

- Completeness means that every POI should have an adequate amount of information. This could also be improved by using templates.

- Logical consistency means that the contributions need to be checked for validity and quality. Moderators can check this information themselves in the portal or users can up-vote and down-vote regarding the quality of a POI. When the quality is not sufficient enough, the app could provide the users with tasks to improve the quality of the information of certain POIs.

- Temporal quality refers to how up-to-date the contributions are. By giving the contributions a time-stamp, moderators or active users can update the information about some POIs when they think it is outdated.

Non-traditional quality factors include: 
- Malicious or mischievous content can be reduced by using user profiles and regular check-ups of POIs by moderators, as well as a voting system.

- Specification with User Participation and Documenting. The data quality of Geo-spatial Metadata is concerned with the quality of attributes, which can be achieved by using templates for users to fill in. However, there should be a balance between the amount of information that is mandatory to fill in and the amount of freedom the user is provided with.

- Linus's Law says that the more people review a problem, the better the solution will be. We suggest that the app has to give the user the option to review the POIs in their neighborhood. This is the area they know the best and will most likely result in the highest quality of information.

- Hierarchical Structures for Quality Assurance refers to using moderators and other hierarchical structures. We suggest that the app should use moderators, a reputation system and ranks. This will be covered in the Gamification section.

\section{Motivators}

Crowd-sourced geo-spatial data has two central parts, i.e., the crowd-sourcers and the geo-spatial data. Much of what is discussed up until now has explained what crowdsourcing exactly is and how the quality of geo-spatial data can be assessed. Yet, maybe the most important part is to look at how people can be attracted to contribute to the app using incentives, understand their motivations for contributing and respond to that, and how to retain these contributors. To help understand what the motivations for participating are and how they are different for the contributors, it is important to first find out which types of people participate in crowdsourcing geo-spatial data [60]. Crowd-sourcers can be categorized as follows [61]:

1. Map lovers and experts who are happy to provide accurate information in cases when maps are wrong or are missing information. These could be retired professional mappers; when they see something wrong on a map, they might be willing to let the authorities know.

2. Casual mappers who can be part of a biking/hiking community and map whilst doing those activities. Casual mappers are most of the time only willing to spend a relatively low effort for mapping and would rather upload new data than looking for errors.

3. Media mappers who respond to specific campaigns such as mapping parties and post-disaster events.

4. Passive mappers who automatically provide information via their mobile phones often without even knowing it. This regards information such as where traffic jams occur.

5. Open mappers who actively contribute to platforms such as OSM. This is by far the largest group, and their number is constantly growing. They are motivated by contributing and using good public data.

6. Paid mappers who are driven by getting paid for doing an activity, e.g., Mechanical Turk from Amazon (a platform that pays users a small amount of money for small tasks).

These categories already provide some insight into the different types of interest in the subject (map lovers, experts, open mappers) or material gain. Although it could be said that a lot of motivation is thought to be altruistic, the range of motivations is much more complex and nuanced [60,61]. Not all of these categories are relevant for the city application, as it attracts other users in comparison with other crowdsourcing applications. Categories that are probably less relevant are casual mappers and passive mappers. The casual mappers do not necessarily apply to the city application because those people mix mapping with other activities, and as we are capturing intangible cultural heritage, this does not mix well with mountain biking or rock climbing. The passive mappers are not relevant to the city application simply because they don't provide intangible cultural heritage data. The other categories are all quite interesting and relevant for our case. Map lovers and experts can be people that know a lot about Utrecht or even their local neighborhood and want that information to be correct. It could also be someone that has worked at a place of interest for a long time and wants the application to display correct information about it. 
Media mappers will probably become active after the city application has been promoted somewhere and they hear about it. Open mappers will hopefully be the biggest group and will consist of people that actually think the idea of the project is interesting and want to contribute to it. Mappers that are motivated by financial incentives can be used when the city application will make use of money to let people perform tasks.

\section{Motivational Factors Specific to Geo-Crowdsourcing}

Geo-crowdsourcing (GeoCS) has demonstrated itself to be a potential problem-solving tool for public management [62]. Budhathoki and Haythornthwaite [63] provided a comprehensive list of different motivational factors that they found in the literature regarding domains for motivation: volunteerism; leisure; and the generation of knowledge online (see Table 1). These factors can also be assigned to two different categories: (1) intrinsic motivation, which comes from from the individual itself, and (2) extrinsic motivation, which is influenced by the outside. Both are very important as they are both positively associated with user participation [64]. For extrinsic motivation, one can think of gaining a positive reputation on the platform after a successful contribution or receiving a financial reward for contributing. Because this list is the most comprehensive one we found, these factors can provide a basis for investigating which motivations the participants of the city application can have.

Using these factors, Budhathoki and Haythornthwaite [63], conducted a survey to try and understand which of these motivational factors were relevant for OSM volunteers. For the survey, they divided 444 OSM volunteers into two groups: serious mappers and casual mappers, based on how much and how often they contributed, and how long they had been contributing. From the survey, they found the most important motivational factors. For both groups, two extrinsic factors, i.e., community and project goal, and two intrinsic factors, altruism, and unique ethos were the most essential factors. Other important factors included: fun, trust in the system, the freedom to provide the information wherever they wanted and local knowledge (instrumentality and self-efficacy). Both groups also had some distinct results. Unique ethos was ranked higher by casual mappers, and learning by serious mappers. Understanding these motivational factors is important for providing strategies to turn casual mappers into serious ones, as serious mappers are more valuable for a participatory platform. Boosting the casual mapper's confidence and emphasizing the importance of local knowledge are two examples of strategies to turn casual mappers into serious ones.

Previous studies [65] have found that the most important factors for OSM and GISCorps volunteers were altruism, personal satisfaction, gaining new geo-spatial knowledge, strengthening of social relationships and fun. Volunteers were also questioned on which kind of incentives they thought would help increase their participation. Many volunteers were interested in additional geo-spatial training. Something that could be used to train volunteers is to use and provide templates, as this is described to be a motivational factor [61]. Using templates, volunteers can learn and train by filling in the templates until they do not need the templates anymore. Composto et al. [66] found that volunteers wanted something back as an incentive for future contributions, such as feedback. When a project or application gives their users feedback regarding their contributions, it may make their users believe that their efforts are recognized and valued. This will prompt users to reciprocate by more actively participating in crowdsourcing tasks and may make them feel indebted to the crowdsourcing project resulting in even more participation [67]. Research has shown that crowdsourcing initiatives with more visible feedback had longer and more sustained participation [68]. 
Table 1. Motivational factors for Crowd-sourced Geo-spatial Data, content adopted from [63].

\begin{tabular}{|c|c|c|}
\hline Type & Factor & Relation to CDG \\
\hline \multirow{13}{*}{ Intrinsic } & Unique ethos & $\begin{array}{l}\text { People think that maps should be openly available and } \\
\text { free for everyone who wants to use it }\end{array}$ \\
\hline & Learning & $\begin{array}{l}\text { Using the application, people gain new knowledge about } \\
\text { mapping, the technologies being used and places }\end{array}$ \\
\hline & Personal enrichment & People find satisfaction in contributing to the project \\
\hline & Self-actualization & $\begin{array}{l}\text { People appreciate their talents, knowledge about local } \\
\text { areas and skills in mapping more after they have } \\
\text { contributed something good. }\end{array}$ \\
\hline & Self-expression & $\begin{array}{l}\text { It enables people to express their knowledge of local areas } \\
\text { and mapping }\end{array}$ \\
\hline & Self-image & $\begin{array}{l}\text { It gives people the opportunity to gain more confidence in } \\
\text { themselves through contributing }\end{array}$ \\
\hline & Fun & $\begin{array}{l}\text { People enjoy the process of contributing and actually } \\
\text { seeing their contribution getting used online }\end{array}$ \\
\hline & Recreation & $\begin{array}{l}\text { Mapping outdoors is a form of recreation that people can } \\
\text { enjoy }\end{array}$ \\
\hline & Instrumentality & $\begin{array}{l}\text { It gives people the opportunity to correct wrong or } \\
\text { incomplete information on a map. }\end{array}$ \\
\hline & Self-efficacy & People feel effective because they contribute to the project \\
\hline & Meeting own needs & $\begin{array}{l}\text { The ability to fill in missing information that is needed for } \\
\text { other applications }\end{array}$ \\
\hline & Freedom of expression & $\begin{array}{l}\text { Ability to provide whatever information people want and } \\
\text { when they want. }\end{array}$ \\
\hline & Altruism & Contribute to a project because it is a social cause. \\
\hline \multirow{11}{*}{ Extrinsic } & Career & $\begin{array}{l}\text { Contributing to a project can actually be mentioned on } \\
\text { people's CVs and can develop skills that can be used on } \\
\text { the market for other jobs and opportunities }\end{array}$ \\
\hline & $\begin{array}{l}\text { Strengthening } \\
\text { relations }\end{array}$ & $\begin{array}{l}\text { Creating social bonds with other participants through } \\
\text { mapping parties and other get-together }\end{array}$ \\
\hline & Project goal & $\begin{array}{l}\text { The goal of the project corresponds to the goals of the } \\
\text { contributor }\end{array}$ \\
\hline & Community & Feeling you belong to an interactive community \\
\hline & Identity & $\begin{array}{l}\text { Becoming part of (another) (sub)-group, e.g., promoting to } \\
\text { a group with a higher level of expertise in mapping and } \\
\text { knowledge }\end{array}$ \\
\hline & Reputation & $\begin{array}{l}\text { Getting recognized for your efforts by the system or } \\
\text { community }\end{array}$ \\
\hline & Monetary Return & Making money by involving yourself in the project \\
\hline & Reciprocity & The idea that if you contribute, others will do the same \\
\hline & System Trust & If the system is trustworthy, it is worth to contribute to \\
\hline & Networking & $\begin{array}{l}\text { Contributing can form networks with people in the } \\
\text { community, or other people that are related to the project, } \\
\text { both locally and internationally }\end{array}$ \\
\hline & Socio-political & $\begin{array}{l}\text { Contributing is in line with people's socio-political } \\
\text { motivations }\end{array}$ \\
\hline
\end{tabular}

\section{Recommendations for a Future-Proof ICH App: The Case of StoryBee}

Based on the literature, we developed recommendations for our ICH app StoryBee, which captures ICH in cities through crowdsourcing. StoryBee is an Android application developed in the context of Utrecht University's Research IT innovation programme, project "Collaborative crowdsourcing tools for sustaining intangible urban heritage". The alpha version of the app was completed in January 2020 and is currently being tested. Below we describe the key design recommendations, which according to 
the literature are essential for the design of a successful mobile ICH application, and which drive the design of StoryBee (Figure 1) .

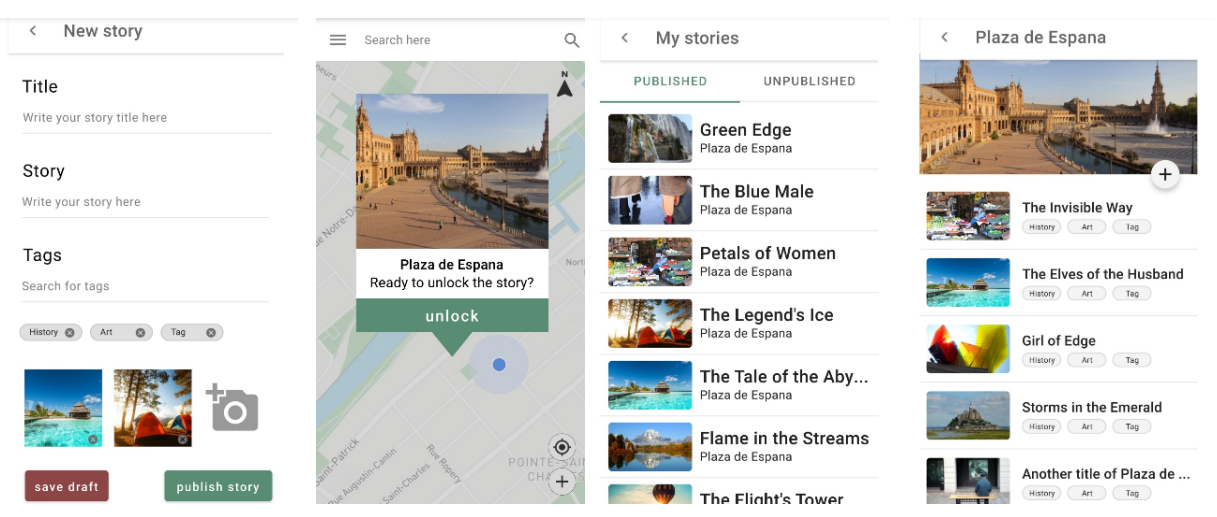

Figure 1. The four paramount features of the StoryBee application design in order: imaging, geo-referencing, contextualization and sharing.

\subsection{Attracting and Recruiting}

User traction is fundamental in designing a successful mobile application; without this element at play, the application life-cycle is compromised. One way to outline and identify the target audience is to interact with some of the users of other existing applications. Gathering user experience surveys, for example, can help yielding valuable insights into the users interests, motivations and other salient usability factors. Only after the target audience is correctly pointed out that the promotion of the framework can be appropriately tailored. Looking into the different ways a cultural application can be sponsored, promotional events fall in the classical route for supporting the cause for attracting and recruiting users, whose personal interests are most likely aligned with the project's objectives.

Media campaigns can also be used to attract new users, despite their reputation for short term retention capabilities. Media campaigns can include advertisements, letting other users share their content on social media and getting featured on TV-shows or other platforms. Mapping parties are also worthy of notice as they are thought to enhance the community spirit and attract not only existing users but also new ones, combining education and entertainment into one single attractive event. Other marketing strategies include the emphasis towards the awareness factor and the promotion of the application amongst Universities, and in so doing, potentially triggering students in the process. Academic engagement would focus on the following: project goal, system trust, personal interest, curiosity, community, networking, altruism, self-efficacy, instrumentality, meeting own needs and personal enrichment.

\subsection{Motivation and Retention}

Attracting users is just one essential aspect when designing a mobile application reliant on its contributors for its development and maintenance. Usability plays a crucial role in assuring that the majority of the users are satisfied and stimulated when returning to the same application. Poor user interfaces can lead to a sharp growth in dissatisfaction and frustration, including unresponsiveness of the interfaces, poor choice of UI components, clashing colour schemes, lack of readability, poor user flow, etc. It is therefore important to list the main criteria to be followed when designing applications for better retention and attraction. Following a list of prescribed measures that we consider indispensable:

Make it a side effect/implicit work. When users are new to the app, they might not necessarily know the set of functionalities at hand. We recommend that the optimal way to introduce the users to the features and functionalities of the framework is to make the act of contributing to the content, a 'side effect' of the user experience. As the application gathers the GPS information of the user, this is capable of recognizing when and if a user is near a particular POI. It can also notify the user when in 
the proximity of POIs. The notification can include information about the POI and where it is exactly, and ask if the user wants to add extra information. This can range from taking a picture, adding a story or simply reviewing other people's contributions. The user can add content to the app without actively going to a certain POI, thus lowering the effort to contribute. This functionality can be optional, so people have the chance to turn the notifications off.

Use Proximity and Familiarity. This is very important and useful because the app works with geo-spatial data. Everyone has a place or neighborhood they know a lot about because they were born there or have lived there for a long time. That is why they think they are an expert with regard to knowing things about those places. They also want the information about their neighborhood to be correct and don't want to see it go extinct. This may even make them feel obligated to add and review content about POI in their neighborhood. The app could ask the user to review POI in his neighborhood and add his own authentic stories and pictures to it. For this to work the user is asked to indicate which area they live in, was born in or knows a lot about. This is not mandatory of course. This is in line with Linus' Law, a quality factor discussed above [59].

Use user-tasks or missions. Up until now, the term task has been used. We would actually suggest that the app uses the term mission, as this has a way less compelling and compulsory sound to it. It is also a term used in a lot of games, which is in line with the gamification mechanics that will be used. The best way to gather geo-spatial data is to actively ask users to perform missions that require them to gather and upload geo-spatial content in a fun way. We recommend having a wide variety of missions, so users don't get bored or feel like they can't do any of the missions provided. Different geo-spatial user tasks and types for data input. For instance, imaging can be achieved by taking a picture of a POI, contextualization is done by adding a story to a specific POI, sharing can be the interaction with other users, validating is achieved by doing a quality check by reviewing and voting on other people's contributions and geo-referencing is done automatically or manually when someone adds a new POI. These missions can be divided over different levels of expertise. When a user has a higher level, quality checks could be done more often for instance, because that user probably has more expertise than lower level users.

It is also important to see the progress of a mission. When the mission is to invite 5 friends to the app, it should have a visual display showing how many people the user has already invited and how many are left. The missions should be clear and small-scale, so users know exactly what to do and do not get bored that easily because it is a small mission. Too many users on one mission should be avoided, as this has a negative impact on participation.

Use a user-profile. As mentioned before, we recommend using a user-profile. Without a user-profile, it is not possible to implement a reputation system, use leader boards or even hand out rewards or points. A user profile is the foundation for all of these mechanics. User profiles can include all sorts of information, ranging from basic personal information to avatars, all of their contributions, their level/rank, reputation, badges and amount of points. The user should be free to decide what he wants to show other people, with a few things being mandatory, such as name and avatar. This also opens up the possibility to have friends on the platform, send each other messages and challenge each other. This positively influences the lineage and reduces the amount of malicious and mischievous content [59]. Making an account should be an option though.

If users want to use the app without an account, that should be possible. You only need an account if you want to participate in any of the activities that require you to have a user-profile, like uploading content, voting, leader-boards and adding friends.

Use a point system. Award points or in-game currency for completing missions. The number of points that can be earned should differ for each mission, based on how difficult it is and how much time it requires. More points can be earned when a user is the first one to visit a certain POI than when the user visits a popular POI. More points can also be earned when a user adds a new POI than when a user just contributes to an already existing POI. Points can be earned by just checking in at POI to increase the number of visits and potential new content. 
These points can be spent on in-game content, like gear for the user's avatar and other features. It is important that points are present throughout the entire application, as this keeps reminding and motivating people.

Use leader boards. Because players can earn points and have a user profile, it is possible to implement one or multiple leader boards. Some users are motivated to rank highly on the leader boards and thus will perform missions to earn points. Leader boards should be short term and have different dimensions. This means that if you have performed two big missions you score higher than someone who has completed two small missions. The benefit of having short term leader boards is that it does not disincentivize users, because they have a new chance of ranking high every week. It is important that the application lets the user know how to rank highly on these leader boards. The top three players on these leader boards could win a reward, or have a chance of winning a bigger award after a longer period of time competing with all winners.

Use competition. Because the app uses points, leader boards, and reputation, there is automatically a competitive element to it. Implementing competitions that users can participate may increase the motivation of some users to participate in the project. Users could invite their friends or other people to a friendly game, which could give them rewards/awards when they win. This competition could be to add as much content as possible in a certain neighborhood in a given time-frame. However, whilst competition should be available, it should also be easy to ignore for those users who are not motivated by competition.

Use a reputation system. As mentioned before, using a reputation system has a lot of benefits and uses. People can up-vote and down-vote other users, and based on those votes get points for their reputation. Being able to vote also makes sure that the quality of contributions is good enough (logical consistency) and it decreases the amount of malicious or mischievous content [59]. Having a better reputation, may mean more influence on new features, more privileges, and even a chance to become a moderator. Having a low reputation may mean restrictions on certain features, such as being unable to vote on and review the quality of other contributions. The app should not disclose every negative ranking or reputation from the user, as this can discourage them to keep using the app. Having a good reputation can result in being recognized in the community and thus will boost the user's confidence. The only way to achieve this is to actively do a lot of missions and have high-quality contributions. This positively influences retention and the quality factor Hierarchical Structures for Quality Assurance [59].

Use rewards. This mechanic has also been mentioned before. Rewards can be monetary or non-monetary. Non-monetary rewards can be in-game content, gear, currency or a big amount of points. Rewards can be earned by winning a competition, ranking high on a leader board or performing unique missions. We suggest that players should not be over-awarded as this might encourage active players to contribute too much and dominate, and thus disincentivizing others from contributing. When monetary rewards are being used, it is important to combine it with other intrinsic motivational factors, as the monetary reward will otherwise be the dominant factor for contributing. This can ultimately lead to users not wanting to contribute anymore without having the chance to earn money.

Use badges. Badges are a type of non-monetary status reward, which people get for achieving something unique. In this case, that could be completing unique missions that require particular skills or much time. People can show badges off on their user profile. This is also a sign of expertise and results in a better reputation. Badges should not be confused with points, as badges are achieved by completing unique missions and are visualized inside the app.

Use feedback. This includes both direct and indirect feedback. Composto et al. [66] found out that volunteers wanted to receive direct feedback from the platform they are using as an incentive for future contributions. When an application or platform gives their users feedback on their contributions, it gives them the feeling they are actually doing something and their efforts are recognized and valued. This will prompt the user to participate more in the future. Ways to do this is to have a good visual display of their POI. So, when a user adds something, they and other users can see the contribution 
right away. We also suggest that the application should highlight a different contribution every day or week on the main page of the app, so everyone can see that contribution. Another way to do this is to have a blog, forum or mail where the newest and best contributions are showcased every week. These emails can also be used to frequently communicate with the users, like talking about new updates or news, which is also good feedback.

Indirect feedback is when a user sees that the contributions of other users are being used and gets motivated by that. Research has shown that crowdsourcing projects that made use of a lot of visible feedback, had longer and more sustained participation [68]. Feedback can also go the other way around. Users should have the option to give the application feedback on what can be better or improved. It is crucial to know the needs of the users to satisfy them [69,70].

Add a timeline to POIs. We suggest that POIs should have a timeline linked to them, so the content that is added to that POI can be linked to a time in the history of that POI. When more people contribute to it and add a timestamp, it can be added to the timeline. 1001 Stories Denmark has implemented a screen for every POI at the same time [71], while we think doing this individually for the POI is a better idea. This increases the Temporal quality of the contributions [59].

Use filters. Because it could be possible to add (Facebook) friends on the application using the user profiles, filters can be used to our advantage. We could give the users the option to only display content that has been added by their friends, thus giving them a more authentic, reliable and trustful experience. The other way around also works. Users can decide to make their contributions only accessible and visible to only certain users (friends). Another filter that can be implemented is to filter on keywords (that other users need to fill in at the template). This way users can choose to only see certain buildings that are interesting to them.

Use collaboration. The fact that the app uses user-profiles and the option to add friends, opens up the possibility to collaborate. Friends could decide to explore a certain POI, smart walk or mission together. Doing things together is always perceived as more fun, so this would be a nice functionality. As is mentioned in the 'use competition' section, users could also challenge each other, like uploading a certain amount of information in a certain time frame.

\section{Discussion}

In general, all results that have been derived from the literature can be used for the application. The features make sure people have the chance to capture and gather their own intangible information and at the same time are motivated to use them. After analyzing the features, it is clear that most of the features try to reduce the effort people have to make to actually use the app and contribute content. Templates, smart walks, making it a side effect, and familiarity and proximity all reduce the amount of time, thoughts and effort the user has to make in order to add content or use the app. Also using gamification has been proven beneficial. On almost every screen of StoryBee some sort of game-element can be seen. Points, leader boards, reputation, and badges make sure the app is fun to use and thus prolonging the retention and continuation of users. Technologies of safeguarding $\mathrm{ICH}$, crowdsourcing methods and system, motivational factors, incentives and methods have all been combined in order to derive a more certain Recommendation that can be used in the production of mobile applications. Especially the mock-ups can help with visualizing and can provide the development team with good ideas of what a cultural application could look like, how the features could be implemented and how certain features could work.

Following the points mentioned in the Section 5, it is important to keep into consideration the four paradigms of ICH preservation: Safety, Quality, Efficacy and Multidisciplinarity, designed for ICH crowd-based applications. The decision to opt for a digital medium when preserving ICH can significantly help the expansion of participation and outreach to other countries, whilst increasing transparency. More so, the digitisation and popularisation of ICH through mobile technology can represent a novel shift of information flow. Going mobile would promote the continuous evolution and 
expansion of $\mathrm{ICH}$, provided that regulators maintain a distinct role and take on a greater responsibility in $\mathrm{ICH}$.

\section{Conclusions}

This research paper aimed to answer two main research questions:

- Which geo-located technological features can help us capture intangible cultural heritage?

- How can people be best motivated to use these features and contribute content?

All features listed in this paper can help to capture ICH, but motivating people to actually use them is another important part of this research. Based on the literature, it can be concluded that incorporating certain game-elements into the app results in the highest amount of contributions and user activity. crowdsourcing systems or applications that use gamification, have a bigger community, longer retention, and overall more contributions.

Using rewards like points, badges, or other compensations is one of the main motivators for people to use certain features, according to the literature. Getting points for adding intangible content, voting on other contributions, interacting with other users and participating in other activities throughout the app helps people to be motivated. Of course, earning points is not that interesting if the user has no way to spend them or show them off. That is why the application should work with customized avatars that people can spend their points on. Badges are earned by performing unique missions and can be shown off to friends or other users.

Another way to show off points and the amount of (good quality) contributions a user has added is to work with reputation. As concluded from the literature review, a lot of successful crowdsourcing sites use a form of reputation, as this increases user engagement and motivation. Feedback is one of the most important motivators for retention and making sure that users keep using an application. Based on the conducted research it can be concluded that almost every application and crowdsourcing system We have reviewed used feedback.

Showing users their own contributions on their user profile, showing them which POI they have visited in the past, highlighting a different contribution every day and allowing them to send messages to the team behind the app are all forms of feedback and are regarded as stimulating in regards to using the features.

Leaderboards is another example of feedback that is also linked to reputation. Having a few short-term leaderboards every week or month that show the users with the most amount of contributions or POI visited gives them exposure and appreciation. At the same time, this motivates other users to contribute more because they want to be in the top 3, especially when they can earn a reward by winning it. All of this interaction results in more user activity and ultimately leads to more contributions. Finally, reflecting on the general principles discussed in Section 5 ("General Principles"), we note several challenges faced by current and future generations, namely challenges of (i) preserving mutating $\mathrm{ICH}$, (ii) choosing the best representative medium for different forms of cultural expression, (iii) ethics and privacy, (iv) ethnocentrism, and (v) interpretability. This paper has covered quite an extensive part of $\mathrm{ICH}$ preservation, and we hope that the design requirements illustrated are taken into consideration as background work for future studies. Overall, in this paper by analyzing prior work, we suggest routes for conceiving, designing and appraising a digital framework that uses crowdsourcing as a way to capture the intangible cultural value of places and support the conservation of the intangible experience, from a user and a community perspective.

Author Contributions: Conceptualization, B.H. and I.L.; Formal analysis, B.H. and I.L.; Methodology, B.H. and I.L.; Resources, B.H. and I.L.; Supervision, I.L.; Visualization, I.L.; Writing-Original draft, B.H. and I.L.; Writing-Review \& editing, B.H., F.L.V., V.-J.K., I.L., K.P. and J.M. All authors have read and agreed to the published version of the manuscript.

Funding: This research received no external funding. 
Acknowledgments: This research has been supported by Utrecht University's "Innovation fund for IT in research", project title "Collaborative crowdsourcing tools for sustaining intangible cultural heritage". The authors also wish to thank the StoryBee software development team.

Conflicts of Interest: The authors declare no conflict of interest.

\section{Abbreviations}

The following abbreviations are used in this manuscript:

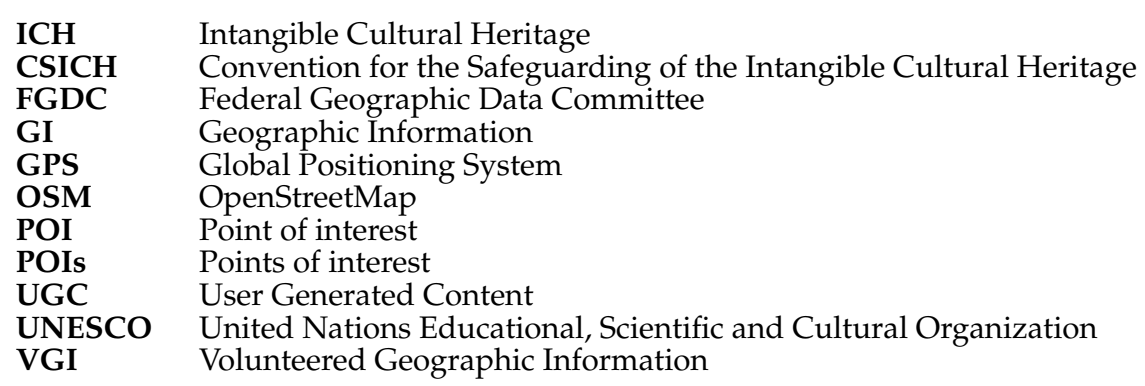

\section{References}

1. UNESCO. Convention for the Safeguarding of the Intangible Cultural Heritage; UNESCO: Paris, France, 2003.

2. Kalay, Y.; Kvan, T.; Affleck, J. New heritage. In New Media and Cultural Heritage; Routledge: London, UK; New York, NY, USA, 2008.

3. Jarvenpaa, S.L.; Lang, K.R. Managing the paradoxes of mobile technology. Inf. Syst. Manag. 2005, 22, 7-23. [CrossRef]

4. Kjeldskov, J.; Graham, C. A review of mobile HCI research methods. In Proceedings of the International Conference on Mobile Human-Computer Interaction, Udine, Italy, 8-11 September 2003; Springer: Cham, Switzerland, 2003; pp. 317-335.

5. Calder, N. Apps: Appropriate, Applicable, and Appealing? In Digital Games and Mathematics Learning; Springer: Cham, Switzerland, 2015; pp. 233-250.

6. Ahmad, Y. The scope and definitions of heritage: From tangible to intangible. Int. J. Herit. Stud. 2006, 12, 292-300. [CrossRef]

7. Vecco, M. A definition of cultural heritage: From the tangible to the intangible. J. Cult. Herit. 2010, 11, 321-324. [CrossRef]

8. Lorusso, S.; Cogo, G.; Natali, A. The Protection and Valorization of Cultural and Environmental Heritage in the Development Process of the Territory. Conserv. Sci. Cult. Herit. 2016, 16, 59-73. [CrossRef]

9. Shirvani Dastgerdi, A.; De Luca, G. Specifying the Significance of Historic Sites in Heritage Planning. Conserv. Sci. Cult. Herit. 2019, 18, 29-39. [CrossRef]

10. Blake, J. On defining the cultural heritage. Int. Comp. Law Q. 2000, 49, 61-85. [CrossRef]

11. Lenzerini, F. Intangible cultural heritage: The living culture of peoples. Eur. J. Int. Law 2011, 22, 101-120.

12. Smith, L.; Akagawa, N. Intangible Heritage; Routledge: London, UK, 2008.

13. Taylor, K. Cultural heritage management: A possible role for charters and principles in Asia. Int. J. Herit. Stud. 2004, 10, 417-433.

14. Czermak, K.; Delanghe, P.; Weng, W. Preserving intangible cultural heritage in Indonesia. In Proceedings of the Conference on Language Development, Language Revitalization and Multilingual Education in Minority Communities in Asia, Bangkok, Thailand, 6-8 November 2003; pp. 1-8.

15. Dimitropoulos, K.; Manitsaris, S.; Tsalakanidou, F.; Nikolopoulos, S.; Denby, B.; Al Kork, S.; Crevier-Buchman, L.; Pillot-Loiseau, C.; Adda-Decker, M.; Dupont, S.; et al. Capturing the intangible an introduction to the i-Treasures project. In Proceedings of the 2014 International conference on computer vision theory and applications (VISAPP), IEEE, Lisbon, Portugal, 5-8 January 2014; Volume 2, pp. 773-781.

16. Mountcastle, A. Safeguarding Intangible Cultural Heritage and the Inevitability of Loss: A Tibetan Example. Stud. Ethnol. Croat. 2010, 22, 339-359. 
17. Shirvani Dastgerdi, A.; Sargolini, M.; Broussard Allred, S.; Chatrchyan, A.; De Luca, G. Climate Change and Sustaining Heritage Resources: A Framework for Boosting Cultural and Natural Heritage Conservation in Central Italy. Climate 2020, 8, 26. [CrossRef]

18. Alivizatou-Barakou, M.; Kitsikidis, A.; Tsalakanidou, F.; Dimitropoulos, K.; Giannis, C.; Nikolopoulos, S.; Al Kork, S.; Denby, B.; Buchman, L.; Adda-Decker, M.; et al. Intangible cultural heritage and new technologies: Challenges and opportunities for cultural preservation and development. In Mixed Reality and Gamification for Cultural Heritage; Springer: Cham, Switzerland, 2017; pp. 129-158.

19. Cominelli, F.; Greffe, X. Intangible cultural heritage: Safeguarding for creativity. City Cult. Soc. 2012, 3, 245-250. [CrossRef]

20. Blake, J. UNESCO's 2003 Convention on Intangible Cultural Heritage: The implications of community involvement in 'safeguarding'. In Intangible Heritage; Routledge: London, UK, 2008; pp. 59-87.

21. Gurel, D.; Çetin, T. An Investigation of Secondary School 7th Grade Students' Awareness for Intangible Cultural Heritage. Online Submiss. 2017, 8, 75-84.

22. UNESCO. Identifying and Inventorying Intangible Cultural Heritage; UNESCO: Paris, France, 2009.

23. Niec, H. Legislative Models of Protection of Cultural Property. Hastings LJ 1975, 27, 1089.

24. Kurin, R. Safeguarding Intangible Cultural Heritage in the 2003 UNESCO Convention: A critical appraisal. Mus. Int. 2004, 56, 66-77. [CrossRef]

25. Aikawa, N. An historical overview of the preparation of the UNESCO International Convention for the Safeguarding of the Intangible Cultural Heritage. Mus. Int. 2004, 56, 137-149. [CrossRef]

26. Brown, M.F. Heritage trouble: Recent work on the protection of intangible cultural property. Int. J. Cult. Prop. 2005, 12, 40-61.

27. Dutfield, G. Protecting Traditional Knowledge and Folklore; International Centre for Trade and Sustainable Development: Geneva, Switzerland, 2003.

28. Bonn, M.; Kendall, L.; McDonough, J. Preserving intangible heritage: Defining a research agenda. Proc. Assoc. Inf. Sci. Technol. 2016, 53, 1-5. [CrossRef]

29. Spiekermann, S. The challenges of privacy by design. Commun. ACM 2012, 55, 38-40. [CrossRef]

30. Spiekermann, S.; Cranor, L.F. Engineering privacy. IEEE Trans. Softw. Eng. 2008, 35, 67-82. [CrossRef]

31. Bélanger, F.; Crossler, R.E. Privacy in the digital age: A review of information privacy research in information systems. MIS Q. 2011, 35, 1017-1042. [CrossRef]

32. DeVries, W.T. Protecting privacy in the digital age. Berkeley Tech. LJ 2003, 18, 283.

33. Safari, B.A. Intangible privacy rights: How Europe's gdpr will set a new global standard for personal data protection. Seton Hall Law Rev. 2016, 47, 809.

34. Kono, T. Intangible Cultural Heritage and Intellectual Property: Communities, Cultural Diversity and Sustainable Development, Intersentia. 2009. Available online: https://papers.ssrn.com/sol3/papers.cfm? abstract_id=1974127 (accessed on 1 January 2009)

35. Paterson, R.K.; Karjala, D.S. Looking beyond intellectual property in resolving protection of the intangible cultural heritage of indigenous peoples. Cardozo J. Int. Comp. Law 2003, 11, 633.

36. Pavlidis, M.; Mouratidis, H.; Gonzalez-Perez, C.; Kalloniatis, C. Addressing privacy and trust issues in cultural heritage modelling. In Proceedings of the International Conference on Risks and Security of Internet and Systems, Mytilene, Greece, 20-22 July 2015; Springer: Cham, Switzerland, 2015; pp. 3-16.

37. Meeker, L. Sounding out Heritage: Cultural Politics and the Social Practice of Quan Ho Folk Song in Northern Vietnam; University of Hawaii Press: Honolulu, HI, USA, 2013.

38. Lixinski, L. Intangible Cultural Heritage in International Law; OUP: Oxford, UK, 2013.

39. Bradley, R. Digital authenticity and integrity: Digital cultural heritage documents as research resources. Portal Libr. Acad. 2005, 5, 165-175. [CrossRef]

40. Garfinkel, S.L. Wikipedia and the Meaning of Truth. Technol. Rev. 2008, 111, 84-86.

41. Meek, S.; Jackson, M.J.; Leibovici, D.G. A flexible framework for assessing the quality of crowdsourced data. In Proceedings of the AGILE'2014 International Conference on Geographic Information Science, Castellón, Spain, 3-6 June 2014; ISBN 978-90-816960-4-3.

42. Zhao, Y.; Campisi, P.; Kundur, D. Dual domain watermarking for authentication and compression of cultural heritage images. IEEE Trans. Image Process. 2004, 13, 430-448. [CrossRef]

43. Coburn, E.; Lanzi, E.; O'Keefe, E.; Stein, R.; Whiteside, A. The Cataloging Cultural Objects experience: Codifying practice for the cultural heritage community. IFLA J. 2010, 36, 16-29. [CrossRef] 
44. Xiao-Chun, L. On Locality and Public Ownership of Intangible Cultural Heritage. J. Guangxi Univ. Natl. Philos. Soc. Sci. Ed. 2008, 3, 2008.

45. Lixinski, L. A tale of two heritages: Claims of ownership over intangible cultural heritage and the myth of 'authenticity'. Transnatl. Disput. Manag. 2014, 11, 1-9.

46. Yang, $\mathrm{H}$. The pros and cons about the digital recording of intangible cultural heritage and some strategies. Int. Arch. Photogramm. Remote Sens. Spat. Inf. Sci. 2015, 40, 461-464. [CrossRef]

47. Giglitto, D. Using Wikis for Intangible Cultural Heritage in Scotland: Suitability and Empowerment. Ph.D. Thesis, University of Aberdeen, Aberdeen, UK, 2017.

48. Miller, T.; Birch, M.; Mauthner, M.; Jessop, J. Ethics in Qualitative Research; Sage: Thousand Oaks, CA, USA, 2012.

49. Morey, T.; Forbath, T.; Schoop, A. Customer data: Designing for transparency and trust. Harv. Bus. Rev. 2015, 93, 96-105.

50. Papangelis, K.; Chamberlain, A.; Liang, H.N. New directions for preserving intangible cultural heritage through the use of mobile technologies. In Proceedings of the 18th International Conference on Human-Computer Interaction with Mobile Devices and Services Adjunct-MobileHCI 16, Florence, Italy, 6-9 September 2016; ACM Press: New York, NY, USA, 2016, doi:10.1145/2957265.2962643.. [CrossRef

51. Garau, C.; Ilardi, E. The "Non-Places" meet the "Places:" Virtual tours on smartphones for the enhancement of cultural heritage. J. Urban Technol. 2014, 21, 79-91. [CrossRef]

52. Pozzebon, A.; Calamai, S. Smart devices for intangible cultural heritage fruition. In Proceedings of the 2015 Digital Heritage, IEEE, Granada, Spain, 28 September-2 October 2015; Volume 1, pp. 333-336.

53. Economou, M.; Meintani, E. Promising beginning? evaluating museum mobile phone apps. In Proceedings of the Rethinking Technology in Museums 2011: Emerging Experiences, Limerick, Ireland, 26-27 May 2011.

54. Garau, C. From territory to smartphone: Smart fruition of cultural heritage for dynamic tourism development. Plan. Pract. Res. 2014, 29, 238-255. [CrossRef]

55. Di Giovine, M. The Heritage Reader. Curator Mus. J. 2011, 54, 363-369. [CrossRef]

56. Dhonju, H.K.; Xiao, W.; Shakya, B.; Mills, J.P.; Sarhosis, V. Documentation of heritage structures through geo-crowdsourcing and web-mapping. In Proceedings of the International Archives of the Photogrammetry, Remote Sensing and Spatial Information Sciences, Wuhan, China, 18-22 September 2017; Copernicus Publications: Gottingen, Germany, 2017; Volume 42, pp. 17-21.

57. Nordmark, S.; Milrad, M. Mobile digital storytelling for promoting creative collaborative learning. In Proceedings of the 2012 IEEE Seventh International Conference on Wireless, Mobile and Ubiquitous Technology in Education, Takamatsu, Japan, 27-30 March 2012; pp. 9-16.

58. Atlas World Heritage Thematic Study on Common Challenges. Available online: http://www. firenzepatrimoniomondiale.it/wp-content/uploads/2018/03/THEMATIC-STUDY-ON-COMMONCHALLENGES.pdf (accessed on 26 February 2020).

59. Rice, M.T.; Paez, F.I.; Mulhollen, A.P.; Shore, B.M.; Caldwell, D.R. Crowdsourced Geospatial Data: A Report on the Emerging Phenomena of Crowdsourced and User-Generated Geospatial Data; Technical Report; George Mason University: Fairfax, VA, USA, 2012.

60. Foody, G.; Fritz, S.; Fonte, C.C.; Bastin, L.; Olteanu-Raimond, A.M.; Mooney, P.; See, L.; Antoniou, V.; Liu, H.Y.; Minghini, M.; et al. Mapping and the Citizen Sensor; Ubiquity Press: London, UK, 2017; pp. 1-12.

61. Heipke, C. Crowdsourcing geospatial data. ISPRS J. Photogramm. Remote Sens. 2010, 65, 550-557. [CrossRef]

62. Haltofová, B. Critical success factors of geocrowdsourcing use in e-government: A case study from the Czech Republic. Urban Res. Pract. 2019, 1-18. doi:10.1080/17535069.2019.1586990 [CrossRef]

63. Budhathoki, N.R.; Haythornthwaite, C. Motivation for open collaboration: Crowd and community models and the case of OpenStreetMap. Am. Behav. Sci. 2013, 57, 548-575. [CrossRef]

64. Zeng, Z.; Tang, J.; Wang, T. Motivation mechanism of gamification in crowdsourcing projects. Int. J. Crowd Sci. 2017, 1, 71-82.

65. Tiwari, R.; Agrawal, A.; Shekhar, S. Contributions of volunteered geographic world: Motivation behind contribution. In Proceedings of the Workshop on Role of Volunteered Geographic Information in Advancing Science, International Conference on Geographic Information Science, Zurich, Switzerland, 14-17 September 2010.

66. Composto, S.; Ingensand, J.; Nappez, M.; Ertz, O.; Rappo, D.; Bovard, R.; Widmer, I.; Joost, S. How to recruit and motivate users to utilize VGI-systems. In Proceedings of the 19th AGILE Conference on Geographic Information Science, Helsinki, Finland, 14-17 June 2016. 
67. Feng, Y.; Ye, H.J.; Yu, Y.; Yang, C.; Cui, T. Gamification artifacts and crowdsourcing participation: Examining the mediating role of intrinsic motivations. Comput. Hum. Behav. 2018, 81, 124-136. [CrossRef]

68. Brito, J.; Vieira, V.; Duran, A. Towards a framework for gamification design on crowdsourcing systems: The GAME approach. In Proceedings of the 12th International Conference on Information Technology-New Generations, IEEE, Las Vegas, NV, USA, 13-15 April 2015; pp. 445-450.

69. Hossain, M. Crowdsourcing: Activities, incentives and users' motivations to participate. In Proceedings of the IEEE 2012 International Conference on Innovation Management and Technology Research, Malacca, Malaysia, 21-22 May 2012; pp. 501-506.

70. À Campo, S.; Khan, V.J.; Papangelis, K.; Markopoulos, P. Community heuristics for user interface evaluation of crowdsourcing platforms. Future Gener. Comput. Syst. 2019, 95, 775-789. [CrossRef]

71. Reestorff, C.M.; Fabian, L.; Fritsch, J.; Stage, C.; Stephensen, J.L. Conjunctions: Introducing cultural participation as a transdisciplinary project. Conjug. Transdiscipl. J. Cult. Particip. 2014, 1, 1-25. [CrossRef]

(c) 2020 by the authors. Licensee MDPI, Basel, Switzerland. This article is an open access article distributed under the terms and conditions of the Creative Commons Attribution (CC BY) license (http://creativecommons.org/licenses/by/4.0/). 